\title{
ON REPRODUCING PROPERTY AND 2-COCYCLES
}

\author{
SAEED HASHEMI SABABE, ALI EBADIAN AND SHAHRAM NAJAFZADEH
}

\begin{abstract}
In this paper, we study reproducing kernels whose ranges are subsets of a $C^{*}$ algebra or a Hilbert $C^{*}$-module. In particular, we show how such a reproducing kernel can naturally be expressed in terms of operators on a Hilbert $C^{*}$-module. We focus on relative reproducing kernels and extend this concept to such spaces associated with cocycles.
\end{abstract}

\section{Introduction}

One of the important and fundamental theories which was developed in recent decades is the theory of reproducing kernels on Hilbert and Banach spaces. Although, the main domain of emersion of this theory raised inside the domain of physics, the development of theory digressed in stochastic process and signal processing. GreenâĂźs functions of selfadjoint ordinary differential equations, the Bergman kernels, the harmonic kernels, and the Szegö kernels are the important examples of reproducing kernels. More interesting properties of reproducing kernel Banach and Hilbert spaces can be found in [3]. Reproducing kernel Hilbert space method provides a rigorous and effective framework for smooth multivariate interpolation of arbitrarily scattered data and for accurate approximation of general multidimensional functions. Moreover, kernels on a semigroup are related to the problem of whether irreversible evolutions of a quantum system can be obtained as restrictions of reversible dynamics in some larger system. Unitary representations of a group $G$ acting on a space $S$ in which the Hilbert $C^{*}$-module is an operator-valued function space on $S$ and the representation is a representation associated by a cocycle on $S \times G$ is studied before by Heo [4].

In the other hand, there are several situations where it is more natural to consider projective representations rather than representations. For instance, in the theory of $C^{*}$-algebras, we can see many results with various kinds of dilations and their covariant versions. There are well-known representation theorems: Naimark-Nagy characterization of positive definite functions on groups and the Stinespring decomposition for completely positive maps

Received August 12, 2017, accepted January 4, 2018.

2010 Mathematics Subject Classification. 47B32, 47A70.

Key words and phrases. Reproducing kernel, 2-semi inner product, 2-semi norm.

Corresponding author: Saeed Hashemi Sababe. 
on a $C^{*}$-algebra. Typical examples for the importance of projective representations are the Bergman spaces on bounded symmetric domains. Upon closer inspection the theories presented in some of past researches generally only apply to simply connected groups. The reason is that the cocycle for the projective representation is assumed continuous, and this property can only be ensured for simply connected groups. However, for general locally compact groups it can be proven that the cocycle can be chosen continuous in a neighborhood of the identity, and for Lie groups the cocycle can even be chosen smooth in a neighborhood of the identity.(see $[1,5]$ )

It should be mentioned that cocycles may be non-continuous, but in the relation of reproducing kernel spaces, we focus on continuous cocycles.

In this paper, we use projective representations on cocycles and 2-cocycles to construct reproducing kernel Hilbert spaces and try to obtain some relations between cocycle based reproducing kernels and 2-cocycle based reproducing kernels.

\section{Preliminaries}

When one considers Hilbert $C^{*}$-modules, one should be rather careful at certain points concerning the existence of the adjoint of operators on a Hilbert $C^{*}$-module and the selfduality of Hilbert $C^{*}$-modules. In the following, we define a Hilbert $\mathscr{A}$-module. The base of this definition comes from right Hilbert $\mathscr{A}$-module but for abbreviation we just call it a Hilbert $\mathscr{A}$-module.

Throughout this article, $S$ and $\mathscr{A}$ denote a nonempty set and a $C^{*}$-algebra, respectively. We denote by $X$ a self-dual Hilbert $\mathscr{A}$-module of $\mathscr{A}$-valued functions on $S$ such that each valuation $f \mapsto f(s) \in \mathscr{A}$ is continuous and linear.

Definition 2.1. A Hilbert $C^{*}$-module over a $C^{*}$-algebra $\mathscr{A}$ is a right $\mathscr{A}$-module $E$ equipped with $\mathscr{A}$-valued inner product $\langle\cdot, \cdot\rangle$ which is conjugate $\mathscr{A}$-linear in the first variable and $\mathscr{A}$ linear in the second variable such that $E$ is a Banach space with respect to the norm $\|x\|=$ $\|\langle x, x\rangle\|^{1 / 2}$.

Example 2.2. Let $X$ be a locally compact Hausdorff space and $H$ a Hilbert space, the Banach space $C_{0}(X, H)$ of all continuous $H$-valued functions vanishing at infinity is a Hilbert $C^{*}$-module over the $C^{*}$-algebra $C_{0}(X)$ with inner product $\langle f, g\rangle(x):=\langle f(x), g(x)\rangle$ and module operation $(f \varphi)(x)=f(x) \varphi(x)$, for all $f \in C_{0}(X, H)$ and $\varphi \in C_{0}(X)$. Every $C^{*}$-algebra $\mathscr{A}$ is a Hilbert $C^{*}$-module over itself with inner product $\langle a, b\rangle:=a^{*} b$.

Definition 2.3. Let $\mathscr{B}$ be a $C^{*}$-algebra. A kernel $k: S \times S \rightarrow \mathscr{B}$ is positive definite if for every $n \in \mathbb{N}$ and $s_{1}, \ldots, s_{n} \in S$ the matrix $\left[k\left(s_{i}, s_{j}\right)\right]_{i, j=1}^{n}$ is positive in $M_{n}(\mathscr{B})$. 
We recall that an element $b$ of a $C^{*}$-algebra $\mathscr{B}$ is called positive if $b=b^{*}$ and $\sigma(b) \subset \mathbb{R}^{+}$. We refer readers to [2] for interesting properties of positive elements and positive operators on $C^{*}$-modules.

It follows from the definition that a kernel $k: S \times S \rightarrow \mathscr{B}$ is positive definite if and only if for all $s_{1}, \ldots, s_{n} \in S$ and $b_{1}, \ldots, b_{n} \in \mathscr{B}$, the sum $\sum_{i, j=1}^{n} b_{i}^{*} k\left(s_{i}, s_{j}\right) b_{j}$ is positive in $\mathscr{B}$. If a kernel $k$ from $S \times S$ into $L_{\mathscr{A}}(X)$ can be written in the form

$$
k(s, t)=v(s)^{*} v(t) \quad \text { for any } s, t \in S,
$$

where $v$ is a map from $S$ to $L_{\mathscr{A}}\left(X, X_{v}\right)$ for some Hilbert $\mathscr{A}$-module $X_{v}$, then $k$ is automatically positive definite. Such a map $v$ is called the Kolmogorov decomposition for $k$. Conversely, every positive definite kernel $k$ with values in $L_{\mathscr{A}}(X)$ has an essentially unique minimal Kolmogorov decomposition.

Consider a Hilbert $\mathscr{A}$-module of $X$-valued functions spanned by those of the form $s \mapsto$ $k(s, t) x$, for some $t \in S$ and some $x \in X$. We denote by $E_{0}$ the set of all $X$-valued functions on $S$ having finite support and by $E$ the set of all $X$-valued functions on $S$. We can identify $E$ with a subspace of the algebraic antidual $E_{0}^{\prime}$ of $E_{0}$ by defining the pairing of $E$ and $E_{0}$ by

$$
(g, f)=\sum_{s \in S}\langle g(s), f(s)\rangle_{X} \quad g \in E, f \in E_{0} .
$$

Given a kernel $k: S \times S \rightarrow L_{\mathscr{A}}(X)$, we can define the associated convolution operator ËIJ $\tilde{k}$ : $E_{0} \rightarrow E$ by

$$
(\tilde{k} f)(s)=\sum_{t \in S} k(s, t) f(t) \quad \text { for every } \quad s \in S .
$$

Then the kernel $k$ is positive definite if and only if its associated convolution operator $\tilde{k} \ddot{E} I J$ : $E_{0} \rightarrow E$ is positive, that is, $(\tilde{k} f, f) \geq 0$ for all $f \in E_{0}$.

For each $s \in S$ and $f \in X$ there exists an element $g_{s} \in X$ such that

$$
f(s)=\left\langle g_{s}, f\right\rangle_{X}
$$

The corresponding reproducing kernel $\kappa: S \times S \rightarrow \mathscr{A}$ is given by

$$
\kappa(s, t)=\left\langle g_{s}, g_{t}\right\rangle_{X} \in \mathscr{A} .
$$

For every $t \in S$ the function $g_{t} \in X$ will be denoted by $\kappa(\cdot, t)$.

Theorem 2.4 (Theorem 3.2 [4]). If a kernel $\kappa: S \times S \rightarrow \mathscr{A}$ is positive definite, then there exists a Hilbert $\mathscr{A}$-module X of $\mathscr{A}$-valued functions on $S$ such that $\kappa$ is the reproducing kernel of X.

We continue our work with an important example. 
Example 2.5. Let $G$ be a locally compact group with a left Haar measure $\mu, \mathscr{A}$ a unital $C^{*}$ algebra, and $X$ a Hilbert $\mathscr{A}$-module. Assume that $G$ is unimodular. We denote by $\mathscr{K}(G, \mathscr{A})$ the right $\mathscr{A}$-module of continuous functions from $G$ to $\mathscr{A}$ with compact supports. For all $f, g$ in $\mathscr{K}(G, \mathscr{A})$, we define an $\mathscr{A}$-valued map $\langle\cdot, \cdot\rangle$ by

$$
\langle f, g\rangle=\int_{G} f(a)^{*} g(a) d \mu(a) .
$$

We denote $L^{2}(G, \mathscr{A})$ by its completion. Let $\pi: G \rightarrow L_{\mathscr{A}}(X)$ be a strongly continuous unitary representation and let $x_{0} \in X$ be fixed. Let $\theta$ be the $\mathscr{A}$-module map from the Hilbert $\mathscr{A}$ module $X$ into the space of $\mathscr{A}$-valued continuous maps defined by

$$
[\theta(x)](a)=\left\langle\pi(a) x_{0}, x\right\rangle_{X} \quad x \in X, a \in G .
$$

It is not hard to see that $[\theta(\pi(a) x)](b)=[\theta(x)]\left(a^{-1} b\right)$ for all $x \in X$ and all $a, b \in G$. Let $Y$ denote the image $\theta(X)$ of $X$ under $\theta$. Assume that $\left\langle x_{0}, x_{0}\right\rangle=I$ and $x_{0}$ is a cyclic unit vector for the representation $\pi$, that is, the closed span of the set $\left\{\pi(a) x_{0} \alpha: a \in G, \alpha \in \mathscr{A}\right\}$ is the whole of $X$. Then the map $\theta: X \rightarrow Y$ is an $\mathscr{A}$-module isomorphism, so that the right $\mathscr{A}$-module $Y$ can be given the structure of a Hilbert A-module isometric to $X$. Indeed, for all $x, y \in X$, we have

$$
\begin{aligned}
\langle\theta(x), \theta(y)\rangle_{Y} & =\int_{G}[\theta(x)](a)^{*}[\theta(y)](a) d \mu(a) \\
& =\int_{G}\left\langle\pi(a) x_{0}, x\right\rangle_{X}^{*}\left\langle\pi(a) x_{0}, y\right\rangle_{X} d \mu(a)=\langle x, y\rangle_{X} .
\end{aligned}
$$

If $G$ is a transformation group on $S$ acting on the right, then there is a canonical action $\pi$ of $G$ on the space of $\mathscr{A}$-valued functions according to the formula

$$
(\pi(a) f)(s)=f(s a) \quad a \in G, s \in S,
$$

where $f: S \rightarrow \mathscr{A}$ is a function. If $X$ is a self-dual Hilbert $\mathscr{A}$-module of $\mathscr{A}$-valued functions on $S$ with a unitary representation $\pi$ of $G$ on $X$, that is, $\pi(a) f \in X$ and $\|\pi(a) f\|=\|f\|$ for all $a \in G$ and $f \in X$, then $X$ is called a $(G, \pi)$-Hilbert $\mathscr{A}$-module.

Definition 2.6. Let $X$ be a Hilbert $\mathscr{A}$-module of $\mathscr{A}$-valued functions on $S$ such that each evaluation $f \mapsto f(s)$ is continuous and $\kappa$ is the reproducing kernel of $X$. Let $G$ be a transformation group on $S$ acting on the right. We denote by $\mathcal{Z}(\mathscr{A})$ the center of $\mathscr{A}$. A continuous function

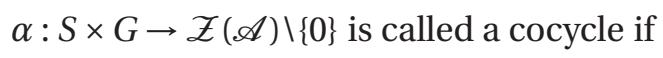

$$
\alpha(s, a b)=\alpha(s, a) \alpha(s a, b) .
$$

for all $a, b \in G$ and $s \in S$. For each cocycle $\alpha$, there is an action $\pi_{\alpha}$ of a group $\mathscr{G}$ on the space of $\mathscr{A}$-valued functions on $S$ such that

$$
\left(\pi_{\alpha}(a) f\right)(s)=f(s, a) \alpha(s, a) .
$$


Let $\alpha: S \times G \rightarrow L_{\mathscr{A}}(X)$ be a cocycle. A kernel $\kappa: S \times S \rightarrow L_{\mathscr{A}}(X)$ is a $G$-kernel associated with $\kappa$ if for all $s, t \in S$ and all $a \in G$

$$
\kappa(s, t)=\alpha(s, a) \kappa(s a, t a) \alpha(t, a)^{*} .
$$

\section{Main results}

In this section, we discus reproducing kernel Hilbert $C^{*}$-modules and 2-inner product version of this kind of spaces. We expand the Kolmogorov decomposition and make a relation between Kolmogorov decomposition and 2-inner product Hilbert $C^{*}$-modules. Finally, we introduce 2-cocycles and explain some of the properties of them. We start with definition of 2-inner product on a Hilbert $C^{*}$-module.

Definition 3.1. Let $X$ be a Hilbert $C^{*}$-module over a $C^{*}$-algebra $\mathscr{A}$ of dimension greater than 1. The function $\langle.,$. . . $\rangle: X \times X \times X \rightarrow \mathscr{A}$ is called a 2-inner product if the following conditions holds,

(1) $\langle x, x ; z\rangle \geq 0$ and $\langle x, x ; z\rangle=0$ iff $x$ and $z$ are linearly dependent.

(2) $\langle x, x ; z\rangle=\langle z, z ; x\rangle$.

(3) $\langle y, x ; z\rangle=\overline{\langle x, y ; z\rangle}$.

(4) $\langle\alpha x, y ; z\rangle=\alpha\langle x, y ; z\rangle$, for all scalars $\alpha \in F$.

(5) $\left\langle x_{1}+x_{2}, y ; z\right\rangle=\left\langle x_{1}, y ; z\right\rangle+\left\langle x_{2}, y ; z\right\rangle$.

Therefore, the pair $(X,\langle., . ;\rangle$.$) is called a 2$-inner product Hilbert $C^{*}$-module.

Example 3.2. Let $C_{0}(X, H)$ be the Hilbert $C^{*}$-module as explained in 2.2. We define a 2-inner product over $C_{0}(X, H)$ by

$$
\langle f, g ; h\rangle=\|h\|^{2} \int \overline{g(x)} f(x) d x-\int \overline{h(x)} f(x) d x . \int \overline{g(x)} h(x) d x .
$$

Definition 3.3. Let $S$ be a nonempty set and $\mathscr{A}$ be a $C^{*}$-algebra. Suppose $X$ is a 2-inner product Hilbert $C^{*}$-module over $\mathscr{A}$ and $\mathscr{X}$ is a subspace of $X$ such that every evaluation $E$ on $\mathscr{X}$ is bounded. In this case, there exists a positive kernel in terms of 2.3 such that for every $x \in S$

$$
E(f)(x)=\left\langle f, k_{x}\right\rangle=f(x) \text { for every } x \in S, f \in \mathscr{X} .
$$

This $\mathscr{X}$ is called a reproducing kernel $\mathscr{A}$-module.

Let $S$ be a set and $\mathscr{F}(S, \mathbb{R})$ be the space of functions $f: S \times S \rightarrow \mathbb{R}$. Clearly, $\mathscr{F}(S, \mathbb{R})$ is an inner product vector space. Let $\mathscr{X}$ be a linear subspace of $\mathscr{F}$ endowed with a 2 -inner product. 
Fix $b \in X$. So we can investigate about $b-C^{*}$-Hilbert module $X_{b}$. Let $\mathscr{X}_{b}$ be a subspace of $X_{b}$ such that all two indexed evaluations

$$
E_{(x, y)}(f)=f(x, y)
$$

be bounded. So by theorem Riesz representation theorem, there exist a function $k_{x, y}$ such that

$$
E_{(x, y)}(f)=f(x, y)=\left\langle f, k_{(x, y)} ; b\right\rangle .
$$

Now we can define a kernel function $K: S \times S \times S \rightarrow \mathbb{R}$ by

$$
K(x, y, z)=\left\langle k_{(x, y)}, k_{(y, z)} ; b\right\rangle .
$$

In this case

$$
K(x, x, x)=\left\langle k_{(x, x)}, k_{(x, x)} ; b\right\rangle=\left\|k_{(x, x)}, b\right\|^{2} .
$$

The pair $\left(\mathscr{X}_{b}, K\right)$ is called a 2 -inner product reproducing $C^{*}$-Hilbert module.

An examples of 2-inner product reproducing kernel Hilbert space is as follows:

Example 3.4. Let $X_{2}^{2}$ be the two variable Hardy-Hilbert space, consists of all analytic functions having power series representations with square-summable complex coefficients and $\mathscr{X}_{I}$ be a subspace of $X_{2}^{2}$ such that

$$
\mathscr{X}_{I}=\left\{f: f\left(z_{1}, z_{2}\right)=\sum_{n=0}^{\infty} a_{n} z_{1}^{n} z_{2}^{n}: a_{1}=0 \text { and } \sum_{n=0}^{\infty}\left|a_{n}\right|^{2}<\infty\right\},
$$

where $I$ denotes the identity function, $f\left(z_{1}, z_{2}\right)=\sum_{n=0}^{\infty} a_{n} z_{1}^{n} z_{2}^{n}$ and $g\left(z_{1}, z_{2}\right)=\sum_{n=0}^{\infty} b_{n} z_{1}^{n} z_{2}^{n}$. The 2 -inner product on $\mathscr{X}_{I}$ is defined by

$$
\langle f, g ; I\rangle=a_{0} \overline{b_{0}}+\sum_{n=2}^{\infty} a_{n} \overline{b_{n}} .
$$

In this case, let $k_{\left(z_{1}, z_{2}\right)}\left(t_{1}, t_{2}\right)=1+\sum_{n=2}^{\infty} \overline{z_{1}^{n} z_{2}^{n}} t_{1}^{n} t_{2}^{n}$, then

$$
f\left(z_{1}, z_{2}\right)=\left\langle f, k_{\left(z_{1}, z_{2}\right)} ; I\right\rangle,
$$

and

$$
K\left(z_{1}, z_{2}, z_{3}\right)=\left\langle k_{\left(z_{1}, z_{2}\right)}, k_{\left(z_{2}, z_{3}\right)} ; I\right\rangle=1+\sum_{n=2}^{\infty} z_{1}^{n} z_{2}^{n}
$$

In the following, we explain some properties of 2-inner product reproducing $\operatorname{kernel} C^{*}$ modules and prove two important theorems.

Proposition 3.5. If $\mathscr{E}^{\dagger}$ is a 2-inner product reproducing kernel $C^{*}$-module on $\mathscr{A}$ with reproducing kernel $k(x, y, z)$, then $k(x, y, x)=\overline{k(y, x, y)}$. 
Proof. By properties of 2-inner product we have

$$
K(x, y, x)=\left\langle k_{(x, y)}, k_{(y, x)} ; b\right\rangle=\overline{\left\langle k_{(y, x)}, k_{(x, y)} ; b\right\rangle}=\overline{K(y, x, y)} .
$$

In the next theorem, we extend Kolmogorov decomposition to 2-inner product reproducing kernel $C^{*}$-modules.

Theorem 3.6. For a positive 2-inner product kernel $k$ from $S \times S \times S$ into $L_{\mathscr{A}}(X)$, there exists a map $v$ from $S$ to $L_{\mathscr{A}}\left(X, X_{v}\right)$ for some Hilbert $\mathscr{A}$-module $X_{v}$ such that

$$
k(x, y, z)=v(x)^{*} v(y) v^{*}(z) \quad \text { for all } \quad z,\|v(z)\| \neq 0 .
$$

Proof. If $f: X \times X \rightarrow \mathscr{A}$ has finite support, define $k f: X \times X \rightarrow \mathscr{A}$ by setting $k f(x, y)=$ $\sum_{z \in S} k(x, y) f(z)$ and denote by $X_{v}^{\prime}$ the set of all these maps $k f$. Clearly, $X_{v}^{\prime}$ is a right module over $\mathscr{A}$ with point wise operations. This space can be naturally endowed with a 2 -inner product

$$
\langle k f, k g, k h\rangle=\sum_{x, y, z \in S}\langle k(x, y, z) f(x), g(y) ; h(z)\rangle .
$$

Let $X_{v}$ be the completion of $X_{0}^{\prime}$.

If $x \in X$, define $v(x): X \rightarrow X_{v}$ by setting $v^{*}(x) f=k(f(x))$. It is easy to check that $v(x)$ is $\mathscr{A}$-linear, bounded and adjointable. So we have

$$
\left\langle v(x)^{*} v(y) v(z)^{*} f, g ; h\right\rangle=\langle k(f(x)), k(f(y)) ; k(f(z))\rangle=\langle k(x, y, z) f, g ; z\rangle,
$$

and therefore

$$
k(x, y, z)=v(x)^{*} v(y) v^{*}(z)
$$

Equation 5 defined an extension of Kolmogorov decomposition. In particular cases, we can choose $v$ as a unitary mapping. The next theorem explains an application of this kind of decomposition.

Theorem 3.7. Let $\mathscr{A}$ be a $C^{*}$-algebra, $X$ be a Hilbert $\mathscr{A}$-module and $\mathscr{X}^{\dagger}$ be a 2 -inner product $\mathscr{A}$ - module. Then there exists a faithful representation $\pi$ of $\mathscr{A}$ on a Hilbert space $X$ and an isometric, linear isomorphism $v$ from $\mathscr{X}^{\dagger}$ onto a concrete Hilbert $\pi(\mathscr{A})$-module $F$ of operators from $X$ to a Hilbert space $Y$ such that

$$
\langle\nu(x), v(y) ; v(z)\rangle=\pi(\langle x, y ; z\rangle) \quad \text { for all } \quad x, y, z \in \mathscr{X}^{\dagger} .
$$


Proof. Let $(X, \pi)$ be any faithful representation of $\mathscr{A}$. Then the kernel, $k: X \times X \times X \rightarrow B(X)$ defined by $(x, y, z) \mapsto \pi(\langle x, y ; z\rangle)$, is positive definite. Hence, the matrix $\pi\left(\left\langle x_{1}, x_{2} ; x_{3}\right\rangle\right.$ is positive in $M n(B(X))$. Since $k$ is positive definite, it admits a Kolmogorov decomposition $U: X \rightarrow$ $B(X, K)$, where $K$ is some Hilbert space. Using the fact that $U(x)^{*} U(y) U(z)^{*}=\pi(\langle x, y ; z\rangle)$ for all $x, y, z \in X$, one easily verifies that $U$ is linear and isometric and that $U(x a)=U(x) \pi(a)$ for all $x \in X$ and $a \in \mathscr{A}$.

Heo defines reproducing kernel associated with cocycles in [4]. In the following, we extend this notion to relative reproducing kernel associated with cocycles.

Definition 3.8. Let $S$ be a non empty set, $\mathscr{A}$ a $C^{*}$-algebra and $X$ a Hilbert $\mathscr{A}$-module such that for any $s, t \in S$ and $f \in L_{\mathscr{X}}$ we have $f \rightarrow f(s)-f(t)$ is continuous. A function $M_{s, t}: S \times S \rightarrow \mathscr{A}$ is called a relative kernel if

$$
f(s)-f(t)=\left\langle f, M_{s, t}\right\rangle \quad \forall f \in L_{\mathscr{A}}(X), s, t \in S .
$$

Theorem 3.9. For any strictly negative definite kernel $M: S \times S \rightarrow L_{\mathscr{A}}(X)$ there exists a unique Hilbert $\mathscr{A}$-module $Y_{M}$ of continuous functions $f: S \rightarrow X$ such that

- $Y_{M}^{0}$ is a dense submodule of $Y_{M}$.

- For each $s \in S$, the evaluation map $E_{s}$ is continuous from $Y_{M}$ to $X$.

Proof. It is easy to check that $Y_{M}^{0}$, the space of all relative kernels is dense on $Y_{M}$. Since the relative kernels construct a basis for this subspace, each evaluation on $Y_{M}$ is bounded and Hence is continues.

The importance of theorem 3.9 is to verification of behavior of representation inherited from $\mathscr{A}$ to $Y_{M}$. In the next theorems we feed back to this subject.

Definition 3.10. Let $G$ be a group and $\alpha: S \times G \rightarrow L_{\mathscr{A}}(X)$ be a cocycle. A relative kernel $M$ : $S \times S \rightarrow L_{\mathscr{A}}(X)$ is a relative $G$-kernel associated with $\alpha$ if for all $s, t \in S$ and all $a \in G$,

$$
M(s, t)=\alpha(s, a) M(s a, t a) \alpha(t, a)^{*} .
$$

Theorem 3.11. For any relative $G$-kernel $M$ associated with a cocycle $\alpha$, the equation

$$
[\pi(a) f](s)=\alpha(s, a) f(s a)
$$


Proof. For a continuous map $\alpha: S \times G \rightarrow L_{\mathscr{A}}(X)$ is a cocycle, then each $\alpha(s, a)$ is invertible. Indeed, for each $s$ and $g$,

$$
\alpha(s, a)^{-1}=\alpha\left(s a, a^{-1}\right)
$$

So for each $f$ we have

$$
\begin{aligned}
\left\langle f, M_{s, t}\right\rangle=f(s)-f(t) & =\pi(a)^{*} \pi(a) f(s)-\pi(a)^{*} \pi(a) f(t) \\
& =\pi(a)^{*} \alpha(s, a) f(s a)-\pi(a)^{*} \alpha(t, a) f(t a) \\
& =\pi(a)^{*}\left\langle\alpha(s, a) f, M_{s a, t a}\right\rangle \\
& =\left\langle\alpha(s, a) f, \pi(a) M_{s a, t a}\right\rangle .
\end{aligned}
$$

Theorem 3.12. Let $M$ be a relative $G$-kernel associated with a cocycle $\alpha$ and let $A$ be an invertible operator in $L_{\mathscr{A}}(X)$. The kernel $M_{A}$ defined by $M_{A}(s, t)=A^{*} M(s, t) A$ is a positive definite relative G-kernel associated with a cocycle $\alpha_{A}$ given by

$$
\alpha_{A}(s, a)=A^{*} \alpha(s, a)\left(A^{-1}\right)^{*} \quad s \in S, a \in G .
$$

Proof. Positiveness of $M_{A}(s, t)$ is a direct corollary of this fact that $M(s, t)$ and $A$ are positive define. Moreover we have

$$
\begin{aligned}
M_{A}(s, t)=A^{*} M(s, t) A & =A^{*} \alpha(s, a) M(s a, t a) \alpha(t, a)^{*} A \\
& =A^{*} \alpha(s, a) M(s a, t a)\left(A^{*} \alpha(t, a)\right)^{*} .
\end{aligned}
$$

In the following, we take an important example which leads us to application of the above theorems.

Example 3.13. Let $X=G$ be a unimodular locally compact group and suppose that $\varphi: G \rightarrow \mathbb{C}$ is a continuous hermitian positive function. It is easy to see that $\varphi$ defines a reproducing kernel Hilbert space. The construction is as follows. Let $C_{0}(G)$ denotes the continuous functions of compact support on $G$. Define

$$
\varphi(G)=\left\{f * \varphi \mid f \in C_{0}(G)\right\}
$$

where

$$
(f * \varphi)(x)=\int f(y) \varphi\left(y^{-1} x\right) d y .
$$

Let $\varphi(f)=f * \varphi$ and define an inner product on $\varphi(G)$ by

$$
\langle\varphi(f), \varphi(g)\rangle=\int \varphi\left(y^{-1} x\right) \overline{f(x)} g(y) d x d y
$$

where $d x$ is Haar measure on $G$. Let $\mathscr{H}(\varphi)$ be the completion of $\varphi(G)$ in the norm comes from above inner product. It is easy to see that in such a way, $\mathscr{H}(\varphi)$ is a relative reproducing Hilbert module. Representation associated to $\mathscr{H}(\varphi)$ is defined as

$$
\left[\pi_{x}(\varphi) f\right](y)=\varphi(f)\left(x^{-1} y\right) .
$$


In this section we define 2-cocycles on a group $G$ and try to extend concepts and relations of cocycles to 2-cocycles. Moreover, we try to find some relations between cocycles and 2cocycles.

Definition 3.14. Let $S$ be a set and $A$ be an abelian group. Let $G$ be a transformation group on $S$ acting on the right. A continuous function $\rho: S \times G \rightarrow A$ is called a 2-cocycle if

$$
\rho(s, g h) \rho(s, h)=\rho(s, g) \rho(s g, h)
$$

for all $g, h \in G$ and $s \in S$.

Clearly for an invertible $\rho$ we have

$$
\rho(s, g)=\rho(s, g h) \rho(s, h) \rho^{-1}(s g, h)
$$

We denote the set of all invertible 2-cocycles by $\operatorname{In} v_{2}(S, G)$.

The concept of 2-cocycles can be related to reproducing kernel spaces in a natural way. The construction is as follows. Let $X$ be a Hilbert $\mathscr{A}$-module of $\mathscr{A}$-valued functions on $S$ such that each evaluation $f \mapsto f(s)$ is continuous and $\kappa$ is the reproducing kernel of $X$. Let $G$ be a transformation group on $S$ acting on the right. The function $\rho$ defined as the equation 7 is a 2-cocycle on a reproducing kernel space. Clearly, $\rho$ belongs to $X$.

Similar to definition of a kernel associated to cocycles, one can extend the definition associated to 2-cocycles.

Definition 3.15. Let $\rho: S \times G \rightarrow L_{\mathscr{A}}(X)$ be a 2-cocycle. A kernel $\kappa: S \times S \rightarrow L_{\mathscr{A}}(X)$ is a $G$-kernel associated with $\rho$ if for all $s, t \in S$ and all $g \in G$,

$$
\kappa(s, t)=\rho(s, g) \kappa(s g, \operatorname{tg}) \rho(t, g)^{*}
$$

Lemma 3.16. Let $\alpha: S \times G \rightarrow L_{\mathscr{A}}(X)$ and $\rho: S \times G \rightarrow L_{\mathscr{A}}(X)$ be a cocycle and 2-cocycle, respectively. If $\rho(s, g h) \rho(s g, h)^{-1}=1$ for all $g, h \in G$ then $\rho(s, g)=\alpha(s, g)$.

Proof. If $\rho(s, g h) \rho(s g, h)^{-1}=1$ then $\rho(s, g h)=\rho(s g, h)$. So by definition of 2 -cocycle we have

$$
\rho(s, g)=1 \text { for all } g, h \in G \text {, }
$$

therefore

$$
\alpha(s, g h) \alpha(s g, h)^{-1}=\rho(s, g h) \rho(s g, h)^{-1} \text { for all } g, h \in G \text {. }
$$

Theorem 3.17. Let $\operatorname{Inv}(S, G)$ and $\operatorname{In} v_{2}(S, G)$ denotes the set of all invertible cocycles and 2cocycles, respectively. then $\operatorname{In} v(S, G) \subset \operatorname{Inv}_{2}(S, G)$. 
Proof. Let $\alpha: S \times G \rightarrow L_{\mathscr{A}}(X)$ be a cocycle. One can easily fined a 2-cocycle $\rho$ such that $\rho(s, g h)=\rho(s g, h)$. In this way, by lemma 3.16, $\alpha(s, g)=\rho(s, g)$ for all $g \in G$ and hence $\alpha \in$ $\operatorname{In} v_{2}(S, G)$.

\section{Acknowledgements}

The best thanks of the first author to professor Un Cig Ji for his kind suggestions.

The first author has to express his appreciation to his wife, Nassim, for standing on his mid-night works.

Authors are so grateful to reviewers for their wise consideration and deep comments and suggestions.

\section{References}

[1] J. G. Christensen, A. H. Darweesh and G. Olafsson, Coorbits for projective representations with an application to Bergman spaces, arXiv:1704.02522v1 [math.FA], 2017.

[2] M. Frank and D.R. Larson, Frames in Hilbert $C^{*}$-modules and $C^{*}$-algebras, J. Operator theory, 48 (2002), 273-314.

[3] S. Hashemi Sababe and Ali. Ebadian, Some properties of reproducing kernel Banach and Hilbert spaces. Sahand Communications in Mathematical Analysis. http: //scma . maragheh.ac.ir/article_27822.html (2017). Accessed 11 October 2017.

[4] J. Heo, Reproducing kernel Hilbert -modules and kernels associated with cocycles, Journal of Mathematical Physics, 49 (2008), 103507-103519.

[5] M. H Hsu and N. C Wong, Inner products and module maps of Hilbert $C^{*}$-modules, Preprints.

Department of Mathematics, Payame Noor University (PNU), P.O. Box, 19395-3697, Tehran, Iran.

E-mail: Hashemi_1365@yahoo.com

Department of Mathematics, Payame Noor University (PNU), P.O. Box, 19395-3697, Tehran, Iran.

E-mail: Ebadian.ali@gmail.com

Department of Mathematics, Payame Noor University (PNU), P.O. Box, 19395-3697, Tehran, Iran.

E-mail: Najafzadeh1234@yahoo.ie 